\section{Specimen Coating for High Resolution Electron Microscopy}

Phil Missing, Emitech, Ltd.

Probably the most common coating technique in electron microscopy is sputter coating, primarily for SEM where a thin electrically conducting film is required to prevent charging and to enhance secondary electron emission.

These systems are relatively inexpensive, and the modern instruments are easy to use, often being fully automated.

\section{D.C. Sputtering}

The direct current (dc) sputter coater uses the arrangement of two electrodes. One positive - the specimen stage, and one negative - the target, typically gold. A glow discharge is formed, and the sputtered material from the target is deposited onto the specimen.

The sputtering efficiency is often enhanced by the use of a magnetron head. An inert gas such as Argon is used, and a suitable low pressure is achieved using a two stage rotary vacuum pump. The grain size for gold target material is of the order of $2-5 \mathrm{~nm}$. To achieve a continuous film, the thickness of deposition is typically $10 \mathrm{~nm}$ but may be somewhat thicker, up to $20 \mathrm{~nm}$ for an irregular specimen.

\section{Chromium Deposition}

With a turbo sputtering system it is now possible to sputter chromium and achieve fine grain size of order of $0.5 \mathrm{~nm}$ thick - which is electrically conductive and continuous. This development in sputter coaters provides ultra thin, high quality films for the users of high resolution SEM.

Until recently, these coatings have been suitable for most SEM applications. With the advent of high resolution SEM, in particular field emission, there is an increasing requirement to improve the performance of the coatings to smaller, continuous films.

While ion beam systems are being employed, the deposition times are long and the instrumentation is relatively expensive

\section{ANALOG/DIGITAL PRINTERS}

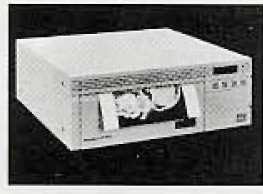

Seikosha Video Printers

- 256 Grev Levels

- $10 \mathrm{MHz}-135 \mathrm{MHz}$

- 5c-19c-40c Hardcopies

- $3 \times 4,81 / 2 \times 8$ or

$81 / 2 \times 11$ Formats

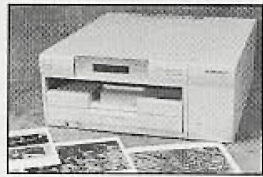

Mitsubishi Printers

- Dye-Sublimation Printers

- Analog/Digital Input

- 16.7 Million Colors

- $4 \times 4$ thru 12 x 18 Format

- Thermal Greyscale Printers

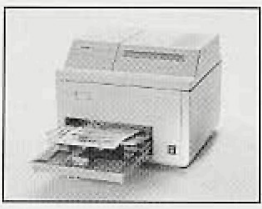

Tektronix Printers

- Dve-Sublimation Printers

- Digital/Postscript Input

- 16.7 Million Colors

- $81 / 2 \times 11$ Thru $81 / 2 \times 14$

- TCP/IP and Ethertalk

VITAL IMAGE TECHNOLOGY INC. OFFERS COMPIETE SALES AND SERVICE,

INCLUDING FREE INSTALLATION IN MOST PARTS OF

THI: UNITED STATES
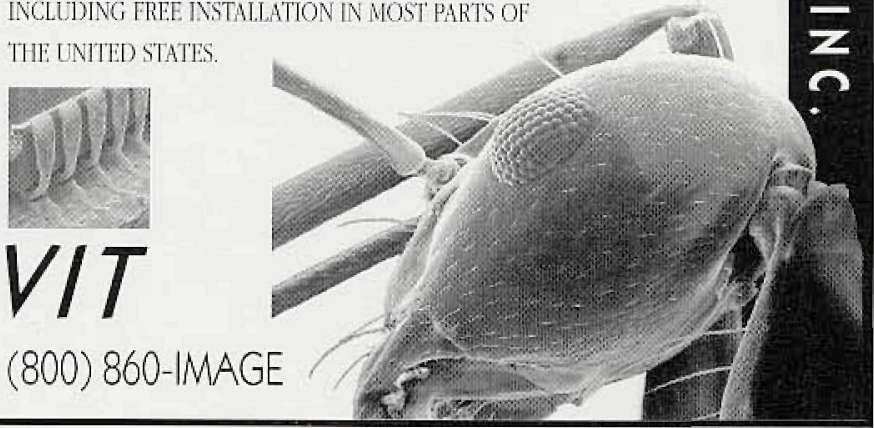

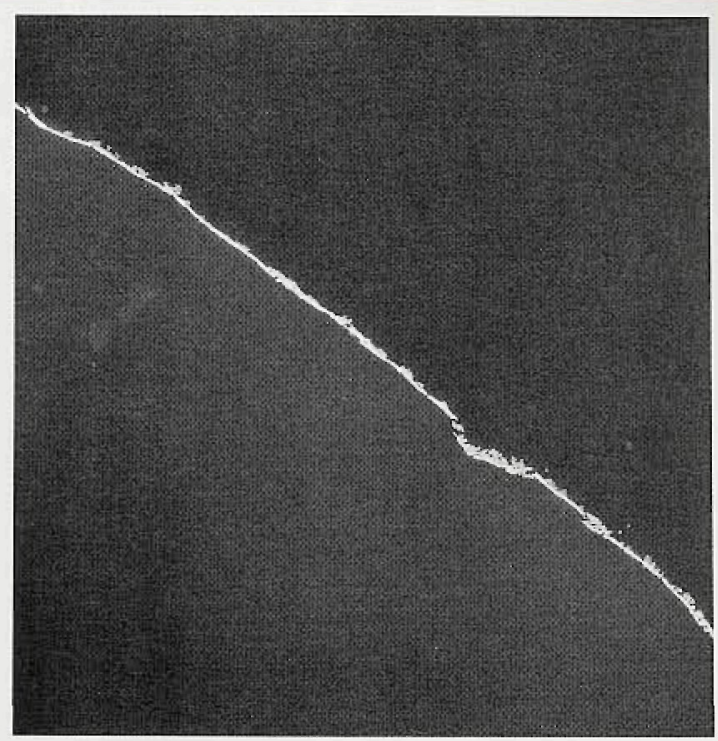

Ultra Thin Continuous Chromium Film

Turbo Sputtering

There are benefits in depositing ultra thin films of chromium on SEM specimens to give improved resolutions at higher magnifications.

To avoid the formation of oxide and contamination when using chromium as the target material, it is necessary to operate at pressures lower than those achievable using two stage rotary vacuum pumps. Such systems usually employ a turbomolecular pumping system.

The system is pumped down to $1 \times 10^{-5} \mathrm{mbar}$. Using an argon bleed, sputtering is typically at $1 \times 10^{-2} \mathrm{mbar}$.

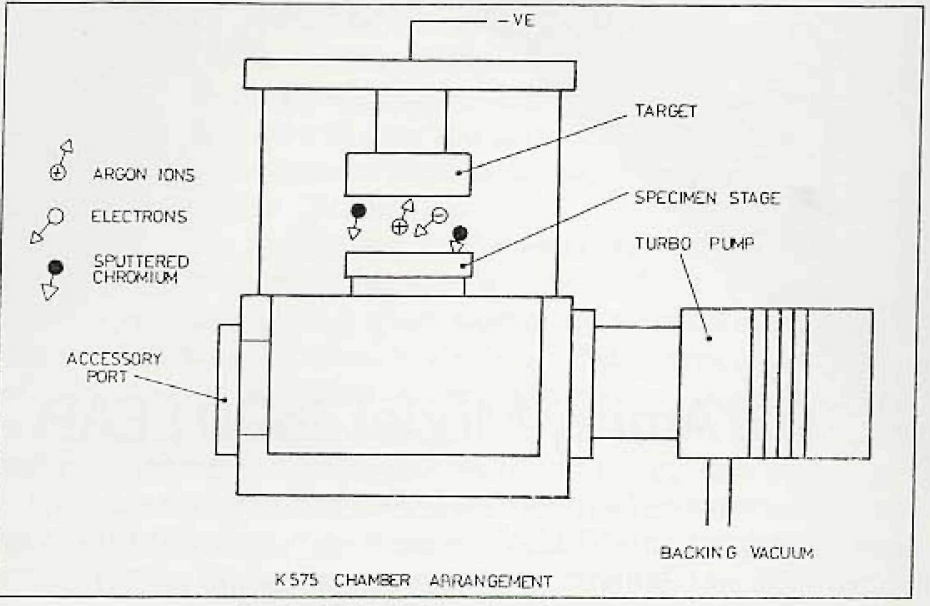

Emitech K575 Chamber Arrangement

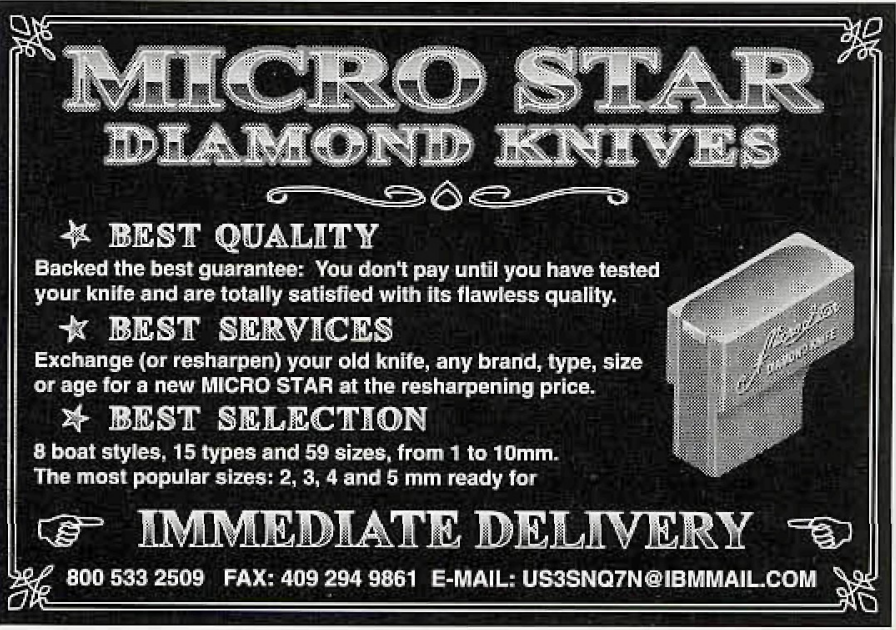




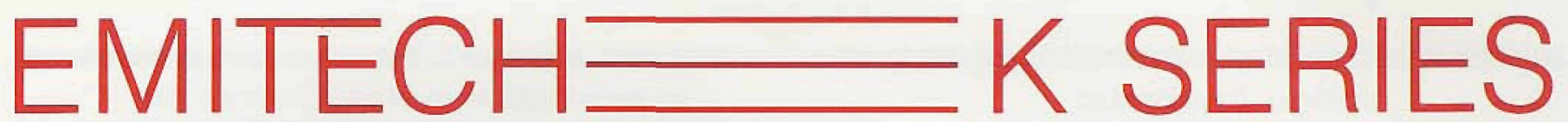

\section{THE COMPLETE 'SPECTRUM' OF PREPARATION EQUIPMENT}
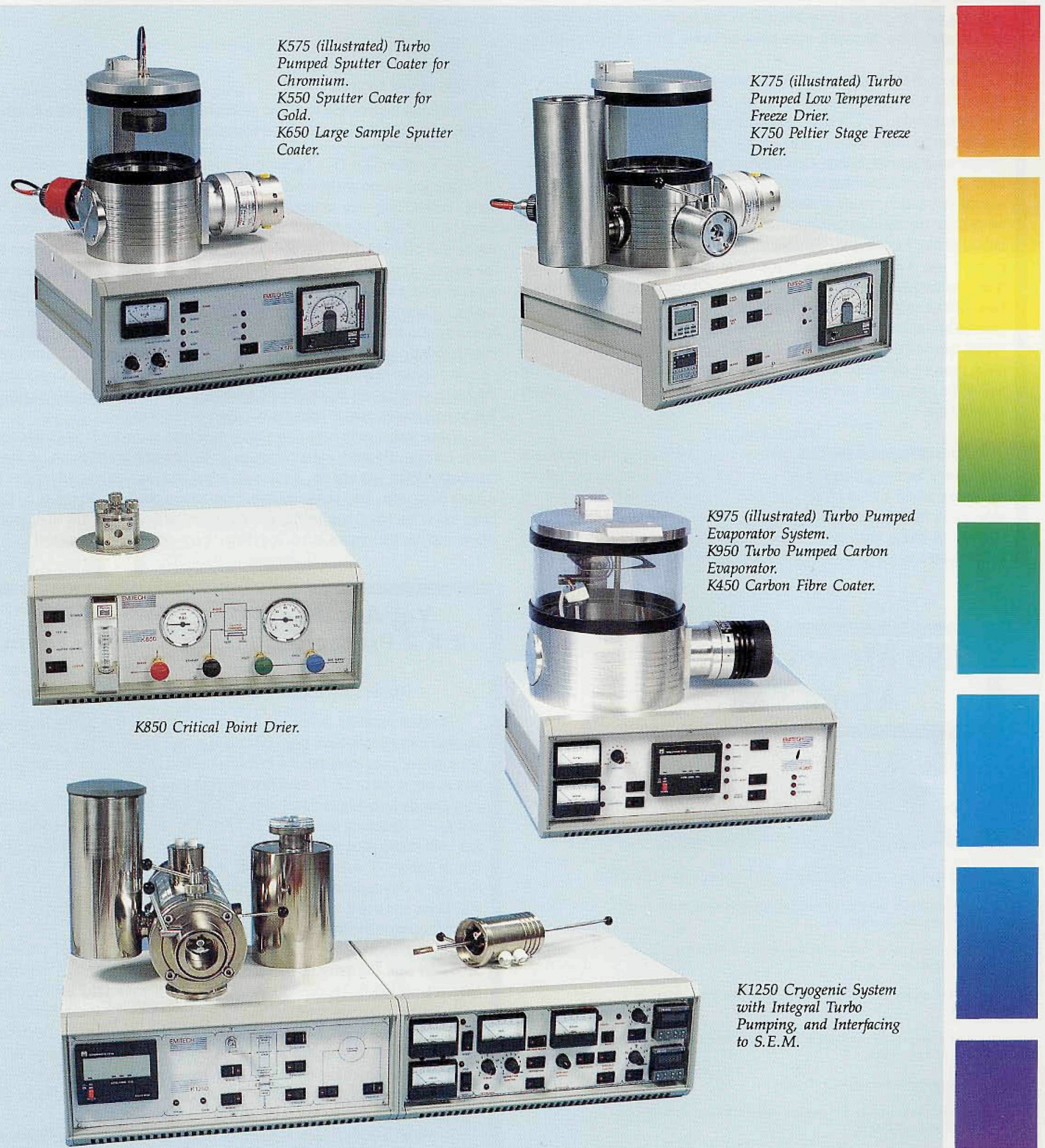

K1250 Cryogenic System with Integral Turbo Pumping, and Interfacing to S.E.M.

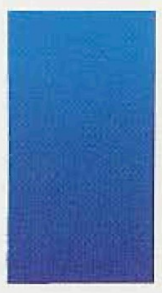

\section{SERVING THE SCIENCE OF ELECTRON MICROSCOPY}

For further datails please contact:

LINDA DAILEY, EMITECH USA, 3845 FM 1960-WEST, SUITE 345, HOUSTON, TEXAS 77068 1-800-444-3137 (713) 893 2067 FAX: (713) 893 8443

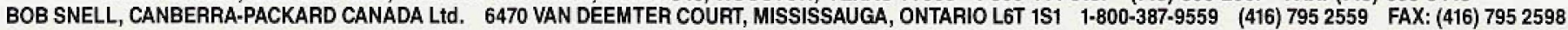

